\title{
Trauma and amyotrophic lateral sclerosis: a case-control study from a population-based registry
}

\author{
E. Pupillo ${ }^{a}$, P. Messina ${ }^{a}$, G. Logroscino ${ }^{b}$, S. Zoccolella ${ }^{b}$, A. Chiò ${ }^{c}, A$. Calvo $^{c}$, M. Corbo $^{d}$, \\ C. Lunetta ${ }^{d}$, A. Micheli $^{\mathrm{a}}$, A. Millul $^{\mathrm{a}}$, E. Vitelli ${ }^{\mathrm{e}}$, E. Beghi $^{\mathrm{a}}$ and EURALS Consortium ${ }^{1}$ \\ ${ }^{\mathrm{a}}$ Laboratorio di Malattie Neurologiche, Istituto Mario Negri, Milan, Italy; ${ }^{\mathrm{b}}$ Clinica Neurologica, Università di Bari, Bari, Italy; ${ }^{\mathrm{c} C l i n i c a}$ \\ Neurologica, Università di Torino, Torino, Italy; ${ }^{\mathrm{d}}$ Fondazione Serena, Centro Clinico NEMO, Hospital Ca' Granda, Milan, Italy; and ${ }^{\mathrm{e}}$ \\ Azienda Ospedaliera della Provincia di Lodi Lodi, Italy
}

\section{Keywords:}

amyotrophic lateral sclerosis, epidemiology, risk factors, trauma

Received 4 January 2012 Accepted 13 March 2012

\begin{abstract}
Background and purpose: Published reports on the association between amyotrophic lateral sclerosis (ALS) and trauma are controversial suggesting the need for a new case-control study done in a large population.

Methods: A case-control study was undertaken in Italy to assess this association. Cases were patients with newly diagnosed ALS from four population-based registries. For each case, two hospital controls were selected, matched for age, sex, and province of residence, one with a neurological (non-degenerative) disease and one with a non-neurological disease (other than orthopedic or surgical). Traumatic events (defined as accidental events causing injuries requiring medical care) were recorded with details on type, site, timing, severity, and complications. The risks were assessed as odds ratios (ORs) with 95\% confidence intervals (CI), crude and adjusted for age, sex, education, interviewee (patient or surrogate), physical activity, smoking, alcohol, and coffee.

Results: The study population comprised 377 patients in each of the three groups. One or more traumatic events were reported by 225 cases $(59.7 \%), 191$ neurological controls $(50.7 \%)$, and 179 non-neurological controls $(47.5 \%)(P<0.01)(\mathrm{OR} 1.63$; 95\% CI $1.25-2.14)(P<0.01)$. The ORs were $3.07(95 \%$ CI $1.86-5.05)$ for patients reporting 3+ traumatic events and 2.44 (95\% CI 1.36-4.40) for severe traumatic events. The ORs remained significant when the analysis was limited to events occurred 5+ and 10+ years before ALS onset, to incident ALS, and direct informant. Conclusion: Antecedent trauma, repeated trauma, and severe trauma may be risk factors for ALS.
\end{abstract}

\section{Introduction}

The role of trauma in amyotrophic lateral sclerosis (ALS) has been repeatedly investigated with conflicting results. Kurland et al. [1] reviewed the early literature and found that retrospective case-control studies gave contradictory results because of selection bias, poor definition of nature and extent of trauma and its chronological relationship to the onset of ALS, and a non-uniform approach to the period preceding the clinical onset of the disease. More rigorously designed studies showed no association of ALS with antecedent

Correspondence: Ettore Beghi, Laboratory of Neurological Disorders, 'Mario Negri' Institute, Via G. la Masa 19, 20156 Milan, Italy (tel.: 390239014542; fax: 390239001916;

e-mail: ettore.beghi@marionegri.it).

${ }^{1}$ All authors in EURALS Consortium present in Appendix. trauma. These observations were confirmed by evidence-based reviews of subsequent reports [2,3].

In recent years, the discussion on traumatic events and ALS has been fueled by reports of several cases amongst Italian professional soccer players [4]. This was confirmed by comparing ALS incidence amongst soccer players and the general population [5]. These findings might be explained by the increased production of reactive oxygen species when combining heavy physical activity, repeated traumatic events, and exposure to toxic contact agents or noxious drugs [6]. However, the association between ALS and physical activity, sport and trauma is still ill-defined [7-10].

We therefore conducted a large case-control study in Italy to assess the association between ALS and trauma. The research hypothesis was whether patients with ALS have a greater chance to have a history of 
trauma, repeated trauma, or severe trauma than the general population.

\section{Material and methods}

The study population included patients with newly diagnosed ALS enrolled in population-based registries of four Italian administrative regions (Lombardia, Piemonte \& Valle D’Aosta, Puglia, Liguria; total population 19997 078; 2009 census) from September 2007 through April 2010. The provision of free or heavily subsidized public medicine in Italy ensures that virtually all patients experiencing ALS symptoms are likely to be visited by a neurologist at some stage during the illness and be hospitalized to ascertain the diagnosis. Newly diagnosed patients with ALS residing in the study regions are then included in the registries, which are part of the European ALS (EURALS) Consortium [11]. The sources of patients with ALS in these registries are multiple and include records from all regional neurology hospital departments, neurophysiology archives, and administrative files. The completeness of case ascertainment has been confirmed for three of four registries (SLALOM for Lombardy, PARALS for Piemonte \& Valle D'Aosta, and SLAP for Puglia) representing $92 \%$ of the entire study population [12-14].

For the purposes of this study, patients were included if they were aged 18 or older and had definite, probable or possible ALS according to the El Escorial (EE) diagnostic classification [15]. For each patient, two hospital controls were selected, matched for age ( \pm 5 years), gender and province of residence. A province is an administrative district (population mean 645 061) within each region, characterized by fairly homogeneous environmental and socio-cultural features. We also tried to match patients and controls by source of interview (patient or surrogate). Informed surrogates were chosen for patients unable to answer and for their matched controls. The first control was from a neurology department and the second from a non-neurological department. We excluded individuals with neurodegenerative disorders (Alzheimer's and Parkinson's disease, Huntington's chorea) and patients in whom hospital admission was related to trauma or orthopedic/surgical illnesses. For patients with ALS, details were collected on the EE diagnostic category, site of onset of symptoms (bulbar, limbs), and duration of symptoms at diagnosis.

After informed consent, each case and control (i.e., the direct informants) or their surrogates underwent a structured interview by a trained investigator. Each interviewer was asked to interrogate the case and his/ her matched control. The following data were recorded in semi-structured questionnaires: date of enrolment, gender, date of birth, years of education, occupation, and physical exercise. Occupation-related physical activity was classified on the basis of the predominant activity and classified as mild, moderate, or strong, based on the interviewee's judgment; the patient or matched control also had to report any sport-related physical activity making him/her breathe hard at least once a month. Smoking, coffee, and alcohol intake were recorded, as ever or never having used tobacco, coffee, or alcoholic beverages on a regular basis for at least 6 months. An accurate history of traumatic events was obtained by direct enquiry. Trauma was defined as any accidental event causing an injury requiring medical care (i.e., any examination by a physician). To identify individuals having incurred in traumatic events, interviewers (one or more for each registry) were instructed to ask the following question: 'Have you ever had any injury requiring medical care...?' Each injury was dated and classified according to cause, severity (based on the patient's report and interviewer's judgment), type, site, and complications.

The study had to be completed after the enrolment of at least 343 cases and 686 controls under the assumption that $1 \%$ of controls and $3.9 \%$ of patients with ALS would report at least one traumatic event requiring hospitalization [odds ratio (OR) 4], with a $5 \%$ level of significance and $80 \%$ power. The percent of individuals in the general population with injuries requiring hospital admission is a conservative estimate derived from a prospective European study (including Italy) on the risk of accidents in epilepsy [16].

The recorded information was transferred from the questionnaires onto a World Wide Web database and centralized for analysis. Missing data were handled using the listwise deletion method. According to this method, an entire record is excluded from analysis if any single value is missing. Descriptive statistics are presented as count and percent, or mean and standard deviation (SD), respectively, for discrete and continuous variables. The chi-Square test was used to compare cases and controls without controlling for confounders (univariate analysis). Multivariate analysis was also carried out using logistic regression models. In each model, data were adjusted for sex, age, education, interviewee (patient/control or surrogate), physical activity (occupational, sport/leisure), smoking, alcohol, and coffee (ever/never). All statistical tests were two-tailed. The results are presented as OR with $95 \%$ confidence intervals $(\mathrm{CI})$.

Eight different logistic regression models were used to evaluate the association between ALS and the following: (A) any traumatic event; (B) number of events 
reported; (C) worst traumatic event reported; (D) number of events requiring hospitalization; (E) number of events leading to disability; (F) site of injury; (G) type of injury; $(\mathrm{H})$ number of injuries by type.

Multivariate analysis was used for all traumatic events and, separately, for events occurred 5+ years before the onset of ALS symptoms (old traumatic events) to lower the possibility to include injuries that might have occurred after the biological onset of the disease. For each control, injuries occurring in the same interval as the companion case were also excluded. Separate analyses were also carried out for men, women, incident ALS (i.e., diagnosis made within the antecedent 12 months), bulbar-onset and limb-onset ALS, traumatic events occurred 10+ years before symptom onset (very old traumatic events), direct informant (after excluding surrogates), site and type of trauma.

As there were no significant differences in the distribution of traumatic events (in general and by number, site, and severity) in the two control groups, neurological and non-neurological controls were combined for multivariate analysis. Owing to the exploratory nature of this study, adjustment of $P$-levels for multiple testing was considered unnecessary [17].

Data were analyzed using the SAS package for PC (SAS Institute, Inc., Cary, NC, USA; 9.1 version). The study was approved by each center's ethics committee and institutional review board.

\section{Results}

A total of 458 patients with ALS and 820 controls (neurological 413; non-neurological 407) were recruited. Eighty-one cases and 66 controls were later excluded for the following reasons: empty questionnaires (52 cases; 28 controls); duplicate input (14 cases; 28 controls); suspected ALS as an EE diagnostic category (eight cases; eight controls); residence outside the study area (three cases; two controls); no matched controls available (four cases). There were 377 eligible patients in each of the three groups. Stroke was the commonest clinical condition for neurological controls $(46.4 \%)$, followed by epilepsy $(17.8 \%)$, peripheral nerve disorders $(9.4 \%)$, headache $(8.6 \%)$, and brain cancer $(8.6 \%)$. Gastrointestinal and liver diseases $(23.7 \%)$ predominated amongst nonneurological controls, followed by cardiovascular disorders $(17.0 \%)$, lung diseases $(17.0 \%)$, tumors $(9.4 \%)$, and diabetes mellitus $(7.0 \%)$.

Definite ALS was recorded in 185 cases $(49.1 \%)$, probable ALS in 151 (40.1\%), and possible ALS in 41 $(10.9 \%)$. The site of onset of symptoms was spinal in 274 patients (72.7\%) and bulbar in $103(27.3 \%)$. Symptom duration at diagnosis was $<12$ months in
222 cases $(58.9 \%)$, between 12 and 24 months in 98 $(26.0 \%)$, and more than 24 months in $48(12.7 \%)$. Symptom duration was not known for nine cases. Table 1 illustrates the general characteristics of the sample. The mean age (SD) was 65.2 (11.3), 65.4 (11.6), and 65.4 (11.4) years for the three groups.

An informed surrogate (generally spouse or sibling) was interviewed on behalf of a quarter of patients with ALS, slightly fewer neurological controls and fewer non-neurological controls $(P<0.05)$. A strong occupational physical activity was reported by slightly more cases than controls. Physically active individuals during sport were fewer amongst patients with ALS $(P<0.01)$. Smoking, alcohol and coffee intakes were significantly less common amongst the cases (Table 1).

Table 2 summarizes the main details of traumatic events. The difference between cases and controls was not confirmed when only old traumatic events were considered. However, cases reported more injuries than both control groups. This was confirmed after excluding events that had occurred within 5 years from symptom onset. Cases also reported more severe

Table 1 Main general characteristics of the sample

\begin{tabular}{|c|c|c|c|}
\hline & $\begin{array}{l}\text { ALS } \\
n=377 \\
n(\%)\end{array}$ & $\begin{array}{l}\mathrm{NC} \\
n=377 \\
n(\%)\end{array}$ & $\begin{array}{l}\mathrm{NNC} \\
n=377 \\
n(\%)\end{array}$ \\
\hline \multicolumn{4}{|l|}{ Sex } \\
\hline Women & $146(38.7)$ & $146(38.7)$ & $146(38.7)$ \\
\hline \multicolumn{4}{|c|}{ Age group (years) } \\
\hline$<65$ & $158(41.9)$ & $174(46.1)$ & $167(44.3)$ \\
\hline $65-74$ & $149(39.5)$ & $125(33.2)$ & $137(36.3)$ \\
\hline$>74$ & $70(18.6)$ & $78(20.7)$ & $73(19.4)$ \\
\hline \multicolumn{4}{|c|}{ Education (years) } \\
\hline $0-4$ & $20(5.3)$ & $31(8.2)$ & $22(5.8)$ \\
\hline $5-7$ & $127(33.7)$ & $124(32.9)$ & $134(35.5)$ \\
\hline $8-12$ & $124(32.9)$ & $118(31.3)$ & $103(27.3)$ \\
\hline $13-17$ & $81(21.5)$ & $77(20.4)$ & $87(23.1)$ \\
\hline$>18$ & $25(6.6)$ & $27(7.2)$ & $31(8.2)$ \\
\hline \multicolumn{4}{|l|}{ Interviewee } \\
\hline Patient & $282(74.8)$ & 308 (81.7)* & $311(82.5)^{* *}$ \\
\hline Relative & $95(25.2)$ & 69 (18.3) & $66(17.5)$ \\
\hline \multicolumn{4}{|c|}{ Physical activity (occupational) } \\
\hline Mild & $101(26.8)$ & $126(33.4)$ & $131(34.7)$ \\
\hline Moderate & $123(32.6)$ & $120(31.8)$ & $106(28.1)$ \\
\hline Strong & $153(40.6)$ & $131(34.7)$ & $140(37.1)$ \\
\hline \multicolumn{4}{|c|}{ Physical activity (leisure/sport) } \\
\hline Yes & $233(61.8)$ & $241(63.9)$ & $271(71.9)^{* *}$ \\
\hline \multicolumn{4}{|l|}{ Smoking } \\
\hline Yes & $174(46.2)$ & $186(49.3)$ & $201(53.3)^{* *}$ \\
\hline \multicolumn{4}{|l|}{ Alcohol } \\
\hline Yes & $166(44.0)$ & $172(45.6)$ & $204(54.1)^{* *}$ \\
\hline \multicolumn{4}{|l|}{ Coffee } \\
\hline Yes & $242(74.7)$ & $291(80.4)$ & $315(85.6)^{* *}$ \\
\hline Missing & 53 & 15 & 9 \\
\hline
\end{tabular}

ALS, amyotrophic lateral sclerosis patients; $\mathrm{NC}$, neurological controls; NNC, non-neurological controls.

${ }^{*} P<0.05 ;{ }^{* *} P<0.01$ (comparisons refer to the ALS group). 
Table 2 Characteristics of traumatic events

\begin{tabular}{|c|c|c|c|c|c|c|}
\hline & \multicolumn{3}{|c|}{ All traumatic events } & \multicolumn{3}{|c|}{ Old traumatic events } \\
\hline & $\begin{array}{l}\text { ALS } \\
n=377 \\
n(\%)\end{array}$ & $\begin{array}{l}\mathrm{NC} \\
n=377 \\
n(\%)\end{array}$ & $\begin{array}{l}\text { NNC } \\
n=377 \\
n(\%)\end{array}$ & $\begin{array}{l}\text { ALS } \\
n=377 \\
n(\%)\end{array}$ & $\begin{array}{l}\text { NC } \\
n=377 \\
n(\%)\end{array}$ & $\begin{array}{l}\text { NNC } \\
n=377 \\
n(\%)\end{array}$ \\
\hline \multicolumn{7}{|c|}{ At least one traumatic event } \\
\hline Yes & $225(59.7)$ & $191(50.7)^{*}$ & $179(47.5)^{* *}$ & $173(45.9)$ & $159(42.2)$ & $151(40.1)$ \\
\hline \multicolumn{7}{|c|}{ Number of events reported } \\
\hline 1 & $129(34.2)$ & $120(31.8)^{*}$ & $115(30.5)^{* *}$ & $104(27.6)$ & $116(30.8)^{*}$ & $109(28.9)^{*}$ \\
\hline 2 & $55(14.6)$ & $47(12.5)$ & $50(13.3)$ & $46(12.2)$ & $32(8.5)$ & $31(8.2)$ \\
\hline $3+$ & $41(10.9)$ & $24(6.4)$ & $14(3.7)$ & $23(6.1)$ & $11(2.9)$ & $11(2.9)$ \\
\hline \multicolumn{7}{|c|}{ Worst traumatic event reported } \\
\hline Mild & $77(20.9)$ & $79(21.1)$ & $84(22.6)^{* *}$ & $59(15.8)$ & 69 (18.4) & $75(20.1)^{* *}$ \\
\hline Moderate & $116(31.4)$ & $92(24.5)$ & $77(20.7)$ & $91(24.3)$ & $73(19.5)$ & $63(16.9)$ \\
\hline Severe & $24(6.5)$ & $18(4.8)$ & $13(3.5)$ & $20(5.4)$ & $15(4.0)$ & $9(2.4)$ \\
\hline Missing & 8 & 2 & 5 & 3 & 2 & 4 \\
\hline \multicolumn{7}{|c|}{ Number of events requiring hospitalization } \\
\hline 1 & $84(22.3)$ & $61(16.2)$ & $56(14.9)^{*}$ & $61(16.2)$ & $59(15.6)$ & $51(13.5)^{*}$ \\
\hline $2+$ & $26(6.9)$ & $21(5.6)$ & $21(5.6)$ & $20(5.3)$ & $8(2.1)$ & $7(1.9)$ \\
\hline \multicolumn{7}{|c|}{ Number of events leading to disability } \\
\hline 1 & $114(30.2)$ & $84(22.3)^{*}$ & $83(22.0)^{*}$ & $93(24.7)$ & $81(21.5)^{* *}$ & $77(20.4)^{* *}$ \\
\hline \multirow[t]{2}{*}{$2+$} & $45(11.9)$ & $36(9.5)$ & $40(10.6)$ & $31(8.2)$ & $12(3.2)$ & $11(2.9)$ \\
\hline & $n$ & $n$ & $n$ & $n$ & $n$ & $n$ \\
\hline \multicolumn{7}{|c|}{ Site of trauma ${ }^{a}$} \\
\hline Head & 58 & 50 & $38^{*}$ & 40 & 28 & 32 \\
\hline Arm & 108 & $73 * *$ & $77^{* *}$ & 84 & $61^{*}$ & 63 \\
\hline Chest & 25 & 20 & 19 & 15 & 16 & 15 \\
\hline Abdomen & 5 & 8 & $14^{*}$ & 4 & 7 & 8 \\
\hline Leg & 112 & 106 & 98 & 85 & 87 & 77 \\
\hline Spine & 24 & 26 & 13 & 17 & 16 & 8 \\
\hline \multicolumn{7}{|c|}{ Type of injury } \\
\hline Concussion & 34 & 22 & $20^{*}$ & 25 & $13^{*}$ & 17 \\
\hline Contusion & 63 & 51 & $32 * *$ & 48 & 37 & $25^{* *}$ \\
\hline Fracture & 133 & $99 * *$ & 108 & 100 & $75^{*}$ & 86 \\
\hline Sprain & 33 & 32 & 28 & 23 & 30 & 22 \\
\hline Strain & 8 & 5 & 4 & 7 & 3 & 3 \\
\hline Wound & 20 & 22 & 23 & 16 & 19 & 18 \\
\hline Other & 17 & 17 & 14 & 12 & 11 & 12 \\
\hline
\end{tabular}

All traumatic events, all events reported; Old traumatic events, events occurring at least 5 years before the onset of symptoms; ALS, amyotrophic lateral sclerosis patients; $\mathrm{NC}$, neurological controls; NNC, non-neurological controls.

${ }^{\mathrm{a}} \mathrm{A}$ traumatic event can involve more than one site and type of injury.

$* P<0.05 ; * * P<0.01$ (comparisons refer to the ALS group).

traumatic events than controls, and more events requiring hospital admission or followed by disability. The commonest site of trauma was the leg, followed by arm and head. Significant differences were found between cases and controls, in decreasing order, for arm, head, abdomen, and spine. Only events involving arms retained significance when the analysis was limited to old traumatic events. Fractures were the commonest injury, followed by contusions and concussions. Each of these injuries predominated in patients with ALS. The association between trauma and ALS was strengthened after multivariate analysis (Table 3).

All ORs were significant. Amongst patients with old traumatic events, the risk was largely limited to men and patients with limb-onset ALS (Table 4).
Significance was retained for history of at least one traumatic event, $3+$ events, moderate or severe events, and $2+$ events requiring hospitalization or leading to disability when multivariate analysis was limited to incident ALS, direct informant (excluding surrogate), and very old traumatic events (see online only Table S1). The analyses were also limited to probable and definite ALS and only to definite ALS; the results obtained were no different from those observed in the whole ALS population (data not shown).

In the multivariate model, significant sites of injury were head (OR 1.59; 95\% CI 1.02-2.47) and arms (OR 1.44; 95\% CI 1.03-2.01), and significant types of injury were concussion (OR 1.85; 95\% CI 1.06-3.24), contusion (OR 1.84; 95\% CI 1.21-2.79), and fractures 
Table 3 Multivariate logistic models

\begin{tabular}{|c|c|c|c|}
\hline Model & Dependent variable & $\begin{array}{l}\text { All traumatic events } \\
\text { ALS }=324^{\mathrm{a}} \\
\text { TC }=724^{\mathrm{b}} \\
\text { OR }(95 \% \mathrm{CI})\end{array}$ & $\begin{array}{l}\text { Old traumatic events } \\
\text { ALS }=324^{\mathrm{a}} \\
\text { TC }=724^{\mathrm{b}} \\
\text { OR }(95 \% \mathrm{CI})\end{array}$ \\
\hline \multirow[t]{3}{*}{ A } & \multicolumn{3}{|c|}{ At least one traumatic event } \\
\hline & No (ref.) & 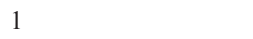 & 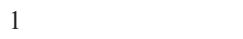 \\
\hline & Yes versus No & $1.63(1.25-2.14)^{* *}$ & $1.33(1.02-1.74)^{*}$ \\
\hline \multirow[t]{5}{*}{ B } & \multicolumn{3}{|c|}{ Number of events reported } \\
\hline & 0 (ref.) & 1 & 1 \\
\hline & 1 & $1.47(1.08-2.00)^{* * * *}$ & $1.09(0.81-1.48)^{* *}$ \\
\hline & 2 & $1.45(0.97-2.18)$ & $1.76(1.14-2.72)$ \\
\hline & $3+$ & $3.07(1.86-5.05)$ & $2.62(1.39-4.91)$ \\
\hline \multirow[t]{5}{*}{$\mathrm{C}$} & \multicolumn{3}{|c|}{ Worst traumatic event reported } \\
\hline & No trauma (ref.) & 1 & 1 \\
\hline & Mild & $1.16(0.81-1.66)^{* * * *}$ & $0.89(0.62-1.30)^{* *}$ \\
\hline & Moderate & $1.96(1.43-2.69)$ & $1.65(1.19-2.29)$ \\
\hline & Severe & $2.44(1.36-4.40)$ & $2.30(1.23-4.32)$ \\
\hline \multirow[t]{4}{*}{$\mathrm{D}$} & \multicolumn{3}{|c|}{ Number of events requiring hospitalization } \\
\hline & 0 (ref.) & 1 & 1 \\
\hline & 1 & $1.77(1.27-2.48)^{* *}$ & $1.29(0.90-1.84)^{* *}$ \\
\hline & $2+$ & $1.40(0.81-2.41)$ & $2.96(1.45-6.04)$ \\
\hline \multirow[t]{4}{*}{$\mathrm{E}$} & \multicolumn{3}{|c|}{ Number of events leading to disability } \\
\hline & 0 (ref.) & 1 & 1 \\
\hline & 1 & $1.68(1.24-2.28)^{* *}$ & $1.35(0.98-1.84)^{* * *}$ \\
\hline & $2+$ & $1.45(0.95-2.21)$ & $3.39(1.90-6.05)$ \\
\hline
\end{tabular}

All models were adjusted for sex, age, education, interviewee, physical activity (occupational or leisure/sport), smoking, alcohol, and coffee. All traumatic events, all events reported; Old traumatic events, events occurring at least 5 years before the onset of symptoms; ALS, amyotrophic lateral sclerosis patients; TC, total controls (neurological controls + non-neurological controls);

ref.= reference category; OR, Odds ratio; $95 \%$ CI, $95 \%$ confidence interval.

$* P<0.05 ; * * P<0.01 ; * * * P<0.001 ; * * * * P<0.0001$.

a 53 missing data because of missing covariates.

$\mathrm{b}_{30}$ missing data because of missing covariates.

(OR 1.39; 95\% CI 1.02-1.89). Furthermore, the OR was 4.77 (95\% CI 1.41-16.13) for a history of $2+$ head injuries and 2.67 (95\% CI 1.17-6.08) for a history of $2+$ arm injuries.

\section{Discussion}

Our findings support an association between ALS and history of traumatic events. Compared to individuals with unrelated clinical conditions, patients with ALS give more frequently a history of trauma, repeated trauma, and/or severe trauma. Causality was supported by time relationship, strength of association, and biological gradient. These findings are in line with other reports [18-25]. However, as the quality of these studies is suboptimal, the possibility that both ALS and trauma could be associated with a common factor cannot be excluded.

The lack of association found by others can be also interpreted in light of methodological inadequacies, including referral bias, different methods of enrolment of cases and controls, recall and interview bias [2629]. Our data are at variance with a European pilot population-based case-control study performed to investigate the association between ALS, physical exercise, trauma and sports [30]. In that study, the proportion of individuals reporting traumatic events was similar amongst cases and controls. However, a higher proportion of patients with ALS (34.4 vs. $24.1 \%$ ) reported repeated traumatic events. Given the small sample size, the study may be underpowered to detect significant differences.

Interestingly, head was the injury site for which the association between (repeated) trauma and ALS was most significant. An association between a history of head trauma and ALS was found by several others [20,23-25]. For participants with multiple head injuries, the risk was more than tenfold [20]. A meta-analysis also indicated a moderately elevated risk of ALS amongst persons with previous head injuries (OR 1.7) [20]. In addition, in the only cohort study of ALS after head injury, comprising 821 individuals who had had a traumatic brain injury and were older than 40 years, there was one patient with ALS (none would have been expected in this small US sample) [25]. 
Table 4 Multivariate logistic models by sex and site of onset (old traumatic events)

\begin{tabular}{|c|c|c|c|c|c|}
\hline \multirow[b]{3}{*}{ Model } & \multirow[b]{3}{*}{ Dependent variable } & \multicolumn{4}{|c|}{ Old traumatic events } \\
\hline & & \multicolumn{2}{|l|}{ Gender } & \multicolumn{2}{|l|}{ Site } \\
\hline & & $\begin{array}{l}\text { Males } \\
\text { ALS }=198 \\
\text { TC }=437 \\
\text { OR }(95 \% \text { CI })\end{array}$ & $\begin{array}{l}\text { Females } \\
\text { ALS }=126 \\
\text { TC }=287 \\
(95 \% \text { CI })\end{array}$ & $\begin{array}{l}\text { Bulbar } \\
\text { ALS }=87 \\
\text { TC }=199 \\
\text { OR }(95 \% \text { CI })\end{array}$ & $\begin{array}{l}\text { Spinal } \\
\text { ALS }=237 \\
\text { TC }=525 \\
\text { OR }(95 \% \text { CI })\end{array}$ \\
\hline \multirow[t]{3}{*}{ A } & \multicolumn{5}{|c|}{ At least one traumatic event } \\
\hline & No (ref.) & 1 & 1 & 1 & 1 \\
\hline & Yes versus No & $1.51(1.07-2.13)^{*}$ & $1.00(0.63-1.60)$ & $1.08(0.64-1.83)$ & $1.44(1.05-1.98)^{*}$ \\
\hline \multirow[t]{5}{*}{ B } & \multicolumn{5}{|c|}{ Number of events reported } \\
\hline & 0 (ref.) & 1 & 1 & 1 & 1 \\
\hline & 1 & $1.28(0.87-1.88)^{*}$ & $0.79(0.46-1.36)$ & $0.71(0.39-1.31)^{* *}$ & $1.28(0.90-1.83)^{*}$ \\
\hline & 2 & $1.92(1.12-3.29)$ & $1.34(0.60-3.00)$ & $3.56(1.56-8.11)$ & $1.38(0.81-2.35)$ \\
\hline & $3+$ & $2.40(1.16-4.97)$ & $4.11(1.01-16.81)$ & $1.26(0.30-5.31)$ & $3.21(1.57-6.57)$ \\
\hline \multirow[t]{5}{*}{$\mathrm{C}$} & \multicolumn{5}{|c|}{ Worst traumatic event reported } \\
\hline & No trauma (ref.) & 1 & 1 & 1 & 1 \\
\hline & Mild & $1.05(0.67-1.65)^{* *}$ & $0.52(0.24-1.09)$ & $0.64(0.30-1.39)$ & $0.95(0.62-1.46)^{* *}$ \\
\hline & Moderate & $1.88(1.24-2.86)$ & $1.45(0.82-2.59)$ & $1.30(0.67-1.51)$ & $1.74(1.19-2.54)$ \\
\hline & Severe & $2.45(1.13-5.30)$ & $1.63(0.50-5.34)$ & $2.15(0.74-6.27)$ & $2.07(0.94-4.59)$ \\
\hline \multirow[t]{4}{*}{$\mathrm{D}$} & \multicolumn{5}{|c|}{ Number of events requiring hospitalization } \\
\hline & 0 (ref.) & 1 & 1 & 1 & 1 \\
\hline & 1 & $1.24(0.80-1.92)$ & $1.33(0.69-2.54)^{*}$ & $1.05(0.51-2.14)$ & $1.38(0.90-2.09)^{*}$ \\
\hline & $2+$ & $2.19(0.93-5.16)$ & $6.47(1.53-27.32)$ & $5.20(1.12-24.16)$ & $2.55(1.12-5.82)$ \\
\hline \multirow[t]{4}{*}{$\mathrm{E}$} & \multicolumn{5}{|c|}{ Number of events leading to disability } \\
\hline & 0 (ref.) & 1 & 1 & 1 & 1 \\
\hline & 1 & $1.60(1.10-2.39)$ & $* * * 0.86(0.48-1.52)$ & $1.14(0.60-2.17)$ & $1.34(0.93-1.93)^{* *}$ \\
\hline & $2+$ & $3.45(1.75-6.78)$ & $3.15(0.94-10.54)$ & $4.19(1.31-13.42)$ & $3.21(1.63-6.32)$ \\
\hline
\end{tabular}

All models were adjusted for $\operatorname{sex}^{\circ}$, age, education, interviewee, physical activity (occupational or leisure/sport), smoking, alcohol, and coffee. ALS, amyotrophic lateral sclerosis patients; TC, total controls (neurological controls + non-neurological controls); ref., reference category; n.s., Not significant; ${ }^{\circ}($ only for bulbar and spinal strata).

$* P<0.05 ; * * P<0.01 ; * * * P<0.001 ; * * * P<0.0001$.

The largest differences between our cases and controls also regarded injuries in the arms, followed by abdomen and spine. An association between injuries to the head, neck, shoulder and/or arm was also found by Gallagher and Sanders [22] in 76\% of patients with ALS and motor axonal injury has been implicated as a possible mechanism based on a number of cases reported during a 14-year period amongst men aged 28 through 43 years at the time of diagnosis [31]. This perhaps explains why trauma has been reported as a risk factor mostly for limb-onset ALS. There are several biological mechanisms by which injuries may trigger the molecular pathways leading to neurodegeneration. They range from inflammatory and glutamate excitotoxicity pathways [32,33], which increase the metabolic demands of neurons and microglia, to oxidative stress pathways that may impact mitochondrial function [34]. In addition, as reported by others [24], the possibility that a specific genotype may modify the effects of injuries on motor neurons cannot be excluded.

Repetitive head injury has also been associated with chronic traumatic encephalopathy, a progressive tau- opathy causing behavioral and personality changes, parkinsonism and dementia [35]. In addition, pathological evidence has been provided with an association between repetitive head trauma in contact sports and the development of motor neuron disease [36]. TDP43 proteinopathy, which is thought to mediate the neuronal cytoskeleton's response to axonal injuries, extended from the frontal and temporal cortex to the spinal cord.

The association between trauma and ALS was apparent only in men. The association between trauma, male gender, and spinal-onset ALS is a possible explanation for the higher incidence of the disease in men with spinal-onset ALS across all ages, which was found in several European countries [12,37]. Men are more likely than women to report traumatic events, repeated injuries, and disabling injuries in their lifetime [38]. In our sample, 152 men (65.8\%) and 73 women $(50.0 \%)$ reported at least one traumatic event; the figures were $126(54.6 \%)$ and $47(32.2 \%)$ only for traumatic events dating back 5 years before the symptom onset. Disabling injuries were reported by 109 men (47.2\%) and 50 women (34.2\%); 92 men (39.8\%) 
and 32 women $(21.9 \%)$ after excluding events within 5 years from symptom onset. However, given the small numbers, the lack of association between injuries, female sex and bulbar-onset ALS may still be to the lack of power to detect significant differences in these subgroups.

Our study has some strength. The major strengths are the representativeness of the ALS population and the large sample size, which may favor the generalizability of the study results. Completeness of case ascertainment is guaranteed by the inclusion of patients with ALS from population-based registries [11]. In addition, 2.0 and $5.3 \%$ of controls and cases, respectively, reported $2+$ traumatic events followed by hospital admission (see Table 2), figures comparable to those expected from the power calculations. Another strength of the study is the characterization of injuries, which were defined according to type, severity, timing, and complications, so we could assess the time relationships, strength, and biological gradient of the purported association.

A number of limitations must be also stressed. First, by definition hospital controls do not represent the general population; however, there is no reason to think that patients with ALS hospitalized for diagnostic purposes and hospital controls come from different source populations In addition, the inclusion of neurological and non-neurological controls, and the variety of acute and chronic disorders are against the possibility that the results could be explained by a single clinical condition and its exposures. Second, this was not a prospective cohort study, so recall bias was unavoidable and no predefined assessment of traumatic events and related injuries was possible. However, we made every effort to collect the required information as accurately as possible using standardized interviews and semi-structured forms. Third, our definition of trauma can be questioned, as mild traumatic injuries might have been excluded. However, this was the only way to identify former accidental events amongst elderly individuals and to collect data less likely to be affected by recall bias. Fourth, classification of the severity of injuries was based on interviewees' subjective opinions. However, erroneous definitions of severity are unlikely to be unevenly distributed in cases and controls. Fifth, data were collected by several investigators and inter-rater reliability is not known. However, the interviewers had been educated to perform a standardized interrogation of patients or surrogates and most cases, and their companion controls were interviewed by the same person. Sixth, more cases than controls were interviewed through an informed surrogate, which may affect recall bias to a different extent. However, the association between traumatic events and ALS was still significant when adjusting for type of informant and when surrogate informants were excluded. Last, the interviewers were not blinded to the diagnosis and the study hypothesis. However, we tried to control for this by asking them to perform structured interviews and record the characteristics of each injury as reported by cases and controls. On the other hand, patients with newly diagnosed ALS may be less likely to be aware of the purported association between the disease and trauma than patients with more advanced disease.

In conclusion, our findings support the theory that previous, repeated and/or severe traumas may be risk factors for ALS. Given the robustness of our findings, confounding and bias seem unlikely. However, a definite answer to this controversial issue could be provided only by a prospective evaluation of a large representative cohort within which exposed subjects (i. e., those reporting traumatic events) are compared to unexposed individuals and followed for a prolonged period of time to identify cases with incident ALS. The organizational and procedural difficulties and the high cost make such a study difficult to perform.

\section{Appendix}

The EURALS Consortium: Coordinator: Ettore Beghi (Milan, Italy)

Steering Commitee: Ammar Al-Chalabi (London, UK); Adriano Chiò (Turin, Italy); Orla Hardiman (Dublin, Ireland); Giancarlo Logroscino (Bari, Italy); Albert Ludolph (Ulm, Germany); Douglas Mitchell (Preston, UK) (deceased); Pierre-Marie Preux (Limoges, France); Robert Swingler (Dundee, Scotland, UK); Leonard van den Berg (Utrecht, The Netherlands).

Members: Elena Alvisi (Pavia, Italy); Monica Bandettini di Poggio (Genua, Italy); Massimiliano Bissolati (Milan, Italy); Paolo Buzzi (Mantua, Italy); Claudia Caponnetto (Genua, Italy); Rosa Capozzo (Bari, Italy); Alberto Cat Berro (Turin, Italy); Cristina Cereda (Pavia, Italy); Mauro Ceroni (Pavia, Italy), Antonietta Citterio (Pavia, Italy); Irene Colombo (Milan, Italy); Massimo Corbo (Milano, Italy); Maria Sofia Cotelli (Brescia, Italy); Luisa De Lodovici (Varese, Italy); Stefania De Mercanti (Turin, Italy); Carlo Ferrarese (Monza, Italy); Vincenza Fetoni (Melegnano, Italy); Massimiliano Filosto (Brescia, Italy); Fabio Formaglio (Milan, Italy); Francesca Gerardi (Milan, Italy); Sara Giacone (Turin, Italy); Giorgia Giussani (Milan, Italy); Maria Cristina Guaita (Legnano, Italy); Antonio Ilardi (Turin, Italy); Lorenzo Lorusso (Chiari, Italy); Christian Lunetta (Milan, Italy); Eleonora Maestri (Milan, Italy); Anna Micheli (Brescia, Italy); Andrea Millul 
(Milan, Italy); Alessandro Padovani (Brescia, Italy); Elena Palazzini (Melegnano, Italy); Michele Perini (Gallarate, Italy); Patrizia Perrone (Legnano, Italy); Alessandro Prelle (Milan, Italy); Alessandra Protti (Milan, Italy); Andrea Rigamonti (Lecco, Italy); Nilo Riva (Milan, Italy); Domenico Santoro (Milan, Italy); Patrizia Secchi (Legnano, Italy); Vincenzo Sidoti (Chiari, Italy); Fiorella Tavernelli (Gallarate, Italy); Lucio Tremolizzo (Monza, Italy); Eugenio Vitelli (Lodi, Italy); Stefano Zoccolella (Bari, Italy).

\section{Acknowledgements}

The study received financial support from the Istituto Superiore di Sanità $(\# 526 \mathrm{D} / 8)$ and from the American ALS Association (ALSA) (\#1524). AC, GL and EB also received funding from the European Union (FP7/ 2007-2013), grant agreement 259867.

\section{Conflict of interest}

Dr Pupillo has no conflicts of interest; Dr Messina has no conflicts of interest; Dr Logroscino has received funding for educational presentation from Novartis and Lundbeck; Dr Zoccolella has no conflicts of interest; Dr Chiò has no conflicts of interest; Dr Calvo has no conflicts of interest; Dr Corbo has no conflicts of interest; Dr Lunetta has no conflicts of interest; Dr Micheli has no conflicts of interest; Dr Millul has no conflicts of interest; Dr Vitelli has no conflicts of interest; Dr Beghi serves on the editorial advisory boards of Epilepsia, Amyotrophic Lateral Sclerosis, Clinical Neurology \& Neurosurgery, and Neuroepidemiology; has received funding for travel and speaker honoraria from UCB-Pharma, Sanofi-Aventis, GSK; and has received funding for educational presentation from GSK.

\section{Supporting Information}

Additional Supporting Information may be found in the online version of this article:

Table S1 Amyotrophic lateral sclerosis and trauma for incident cases, direct informant and very old traumatic events.

Please note: Wiley-Blackwell are not responsible for the content or functionality of any supporting materials supplied by the authors. Any queries (other than missing material) should be directed to the corresponding author for the article.

\section{References}

1. Kurland LT, Radhakrishnan K, Smith GE, et al. Mechanical trauma as a risk factor in classic amyo- trophic lateral sclerosis: lack of epidemiologic evidence. J Neurol Sci 1992; 113: 133-143.

2. Armon C. An evidence based medicine approach to the evaluation of the role of exogenous risk factors in sporadic amyotrophic lateral sclerosis. Neuroepidemiology 2003; 22: 217-228.

3. Armon C. Sports and trauma in amyotrophic lateral sclerosis revisited. J Neurol Sci 2007; 262: 45-53.

4. Beretta S, Carrì MT, Beghi E, et al. The sinister side of Italian soccer (Reflection and Reaction). Lancet Neurol 2003; 2: 656-657.

5. Chiò A, Benzi G, Dossena M, et al. Severely increased risk of amyotrophic lateral sclerosis among Italian professional football players. Brain 2005; 128(Pt 3): 472-476.

6. McArdle A, Pattwell D, Vasilaki A, et al. Contractile activity-induced oxidative stress: cellular origin and adaptive responses. Am J Physiol Cell Physiol 2001; 280: C621-C627.

7. Belli S, Vanacore N. Proportionate mortality of Italian soccer players: is amyotrophic lateral sclerosis an occupational disease? Eur J Epidemiol 2005; 20: 237-242.

8. Valenti M, Pontieri F, Conti F, et al. Amyotrophic lateral sclerosis and sports: a case control study. Eur $J$ Neurol 2005; 12: 223-225.

9. Veldink JH, Kalmijn S, Groeneveld GJ, et al. Physical activity and the association with sporadic ALS. Neurology 2005; 64: 241-245.

10. Scarmeas N, Shih T, Stern Y, et al. Premorbid weight, body mass, and varsity athletics in ALS. Neurology 2002; 59: 773-775.

11. Beghi E, Logroscino G, Chiò A, et al. The epidemiology of ALS and the role of population-based registries. Biochem Biophys Acta 2006; 1762: 1150-1157.

12. Beghi E, Millul A, Micheli A, et al. Incidence of ALS in Lombardy, Italy. Neurology 2007; 68: 141-145.

13. Piemonte and Valle d'Aosta Register for Amyotrophic Lateral Sclerosis (PARALS). Incidence of ALS in Italy. Evidence for a uniform frequency in Western countries. Neurology 2001; 56: 239-244.

14. Logroscino G, Beghi E, Zoccolella S, et al. Incidence of amyotrophic lateral sclerosis in southern Italy: a population-based study. J Neurol Neurosurg Psychiatry 2005; 76: 1094-1098.

15. WFN Research Group on Neuromuscular Disorders. El Escorial World Federation of Neurology criteria for the diagnosis of amyotrophic lateral sclerosis. J Neurol Sci 1994; 124(suppl): 96-107.

16. Van den Broek M, Beghi E. for the RESt-1 Group. Accidents in patients with epilepsy: types, circumstances, and complications: a European cohort study. Epilepsia 2004; 45: 667-672.

17. Bender R, Lange S. Adjusting for multiple testing when and how? J Clin Epidemiol 2011; 54: 343-349.

18. Binazzi A, Belli S, Uccelli R, et al. An exploratory casecontrol study on spinal and bulbar forms of amyotrophic lateral sclerosis in the province of Rome. Amyotroph Lateral Scler 2009; 10: 361-369.

19. Chancellor AM, Slattery JM, Fraser H, et al. Risk factors for motor neuron disease: a case-control study based on patients from the Scottish Motor Neuron Disease Register. J Neurol Neurosurg Psychiatry 1993; 56: 1200-1206.

20. Chen H, Richard M, Sandler DP, et al. Head injury and amyotrophic lateral sclerosis. Am J Epidemiol 2007; 166: 810-816. 
21. Gawel M, Zaiwalla Z, Rose FC. Antecedent events in motor neuron disease. J Neurol Neurosurg Psychiatry 1983; 46: 1041-1043.

22. Gallagher JP, Sanders M. Trauma and amyotrophic lateral sclerosis: a report of 78 patients. Acta Neurol Scand 1987; 75: 145-150.

23. Kondo K, Tsubaki T. case-control studies of motor neuron disease: association with mechanical injuries. Arch Neurol 1981; 38: 220-226.

24. Schmidt S, Kwee LC, Allen KD, et al. Association of ALS with head injury, cigarette smoking and APOE genotypes. J Neurol Sci 2010; 291: 22-29.

25. Williams DB, Annegers JF, Kokmen E, et al. Brain injury and neurologic sequelae: a cohort study of dementia, parkinsonism, and amyotrophic lateral sclerosis. Neurology 1991; 41: 1554-1557.

26. Turner MR, Abisgold J, Yeates DG, et al. Head and other physical trauma requiring hospitalisation is not a significant risk factor in the development of ALS. $J \mathrm{Neu}$ rol Sci 2010; 288: 454-458.

27. Qureshi MM, Hayden D, Urbinelli L, et al. Analysis of factors that modify susceptibility and rate of progression in amyotrophic lateral sclerosis (ALS). Amyotroph Lateral Scler 2006; 7: 173-182.

28. Murros K, Fogelholm R. Amyotrophic lateral sclerosis in Middle-Finland: an epidemiological study. Acta Neurol Scand 1983; 67: 41-47.

29. Cruz DC, Nelson LM, McGuire V, et al. Physical trauma and family history of neurodegenerative diseases in amyotrophic lateral sclerosis: a population-based casecontrol study. Neuroepidemiology 1999; 18: 101-110.
30. Beghi E, Logroscino G, Chiò A, et al. Amyotrophic lateral sclerosis, physical exercise, trauma and sports: results of a population-based pilot case-control study. Amyotroph Lateral Scler 2010; 11: 289-292.

31. Riggs JE, Hobbs GR. Motor axonal injury and amyotrophic lateral sclerosis: risk assessment using a reverse probability analysis technique. Mil Med 2003; 168: 143-145.

32. Lenzlinger PM, Morganti-Kossmann MC, Laurer HL, et al. The duality of the inflammatory response to traumatic brain injury. Mol Neurobiol 2001; 24: 169-181.

33. Arundine M, Tymianski M. Molecular mechanisms of glutamate-dependent neurodegeneration in ischemia and traumatic brain injury. Cell Mol Life Sci 2004; 61: 657-668.

34. Frantseva M, Perez Velazquez H, Tonkikh A, et al. Neurotrauma/neurodegeneration and mitochondrial dysfunction. Prog Brain Res 2002; 137: 171-176.

35. McKee A, Cantu R, Nowinski C, et al. Chronic traumatic encephalopathy in athletes: progressive tauopathy after repeated head injury. $J$ Neuropathol Exp Neurol 2009; 68: 709-735.

36. McKee AC, Gavett BE, Stern RA, et al. TDP-43 proteinopathy and motor neuron disease in chronic traumatic encephalopathy. J Neuropathol Exp Neurol 2010; 69: 918-929.

37. Logroscino G, Traynor BJ, Hardiman $\mathrm{O}$, et al. Incidence of amyotrophic lateral sclerosis in Europe. $J \mathrm{Neu}$ rol Neurosurg Psychiatry 2010; 81: 385-390.

38. Alexandrescu R, O'Brien SJ, Lecky FE. A review of injury epidemiology in the UK and Europe: some methodological considerations in constructing rates. $B M C$ Public Health 2009; 9: 226. 